\title{
Instability mechanisms and transition scenarios of spiral turbulence in Taylor-Couette flow.
}

\author{
Alvaro Meseguer, ${ }^{1, *}$ Fernando Mellibovsky, ${ }^{1}$ Marc Avila, ${ }^{2}$ and Francisco Marques ${ }^{1}$ \\ ${ }^{1}$ Departament de Física Aplicada, Universitat Politècnica de Catalunya, 08034, Barcelona, Spain \\ ${ }^{2}$ Max Planck Institute for Dynamics and Self-Organization, 37073, Göttingen, Germany
}

(Dated: October 5, 2009)

\begin{abstract}
Alternating laminar and turbulent helical bands appearing in shear flows between counterrotating cylinders are accurately computed and the near-wall instability phenomena responsible for their generation identified for the first time. The computations show that this intermittent regime can only exist within large domains and that its spiral coherence is not dictated by endwall boundary conditions. A supercritical transition route, consisting of a progressive helical alignment of localised turbulent spots, is carefully studied. Subcritical routes disconnected from secondary laminar flows have also been identified.
\end{abstract}

PACS numbers: 47.20.-k, 47.20.Lz, 47.27.Cn

A comprehensive understanding of turbulent phenomena necessarily requires a previous explanation of the mechanisms that mediate between laminar and fully disordered fluid motion. One of the most challenging shear flow problems is the understanding of laminar-turbulent coexistence phenomena or intermittency, i.e., spatio-temporal coexistence between laminar and turbulent regions in a fluid flow. Canonical shear flows such as plane Couette flow between inertially countersliding parallel plates or pipe flow in a very long straight pipe of circular cross section exhibit localised turbulence as a prelude to fully developed turbulent flow [1-6]. Open shear flows share many common drawbacks when studying the long term behaviour of turbulent or intermittent regimes, since localised turbulent spots are often advected downstream and leave the domain. Computation of these flows usually assumes streamwise periodicity, overlooking the real boundary conditions at the entrance and exit of the domains and potentially leading to artificial interaction of the leading and trailing edges of localised turbulent spots. A naturally streamwise-periodic problem such as the Taylor-Couette system between independently rotating concentric cylinders solves these difficulties. Furthermore, while transition in open shear flows is typically subcritical, i.e., bypassing linear stability, Taylor-Couette flow exhibits a huge variety of secondary supercritical steady, time periodic, or almost periodic laminar flows before an eventual transition to chaotic regimes [7]. This enables to study transition in a supercritical setting, along with degeneration into subcriticality. We refer the reader to standard monographs and references therein $[8,9]$.

Laminar-turbulent coexistence in Taylor-Couette flow was originally reported by Coles and Van Atta in the 1960s $[10,11]$. They observed interlaced laminar-turbulent helical patterns (see Fig. 1a) so called spiral turbulence or barber pole turbulence, according to Feynman [12]. This pattern has been studied experimentally by many authors later in the 1980s [7, 13] and during the current decade [1, 14]. Spiral turbulence, henceforth termed as SPT, may exhibit hysteretic subcritical behaviour, being sustained even in situations where linear theory predicts stability of the base laminar flow. Linear non-modal analysis has shown a strong correlation between the hysteretic effects of SPT and transient growth of infinites-

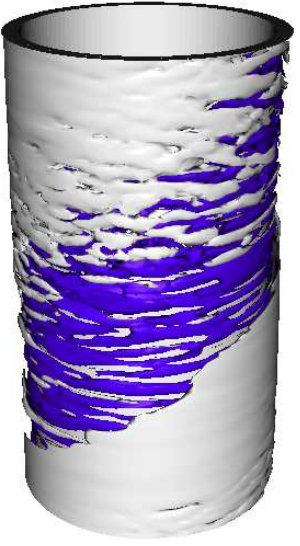

(a)

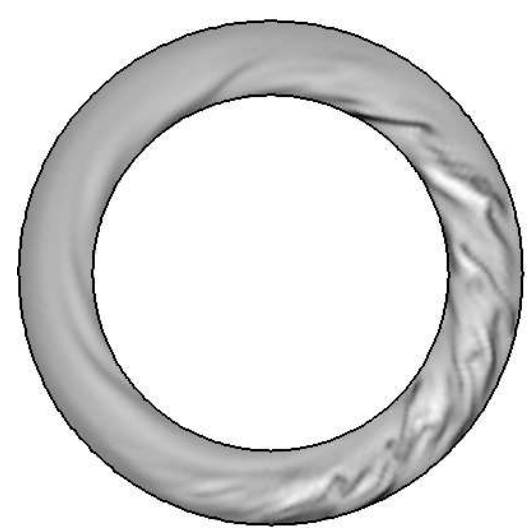

(b)
FIG. 1: Spiral turbulence between counterrotating concentric cylinders (outer cylinder rotating clockwise). (a) Three dimensional view of angular momentum distribution. (b) Annular cross section of axial vorticity distribution. The structure rotates clockwise (see film).

imal perturbations of the basic flow [15]. Numerical simulations carried out in the 1990s identified a secondary instability mechanism apparently responsible for bursting phenomena in counter-rotating Taylor-Couette flow, although the computational aspect ratio used was still too small to capture long range spatial intermittency [16]. More recent nonlinear computations of counter-rotating Taylor-Couette flow have provided new families of subcritical spirals although these structures have a much shorter axial wavelength than the SPT[17]. Recent experiments have addressed the similarities between SPT and other intermittent regimes appearing in plane Couette and Taylor-Couette flows [1]. On those lines, recent numerical explorations have identified parameter ranges in the narrow gap limit of Taylor-Couette flow where some of the characteristics of the plane Couette flow can be recovered [18].

In this Letter the first successful computation of SPT between counterrotating cylinders is reported. It is shown that SPT can only exist in a large enough apparatus and that it is not a byproduct of endwall effects emanating from the top and bottom lids. The simulations also reveal that the instability 
mechanism of SPT is based on a breakdown of vorticity filaments detaching from the inner cylinder. For moderate speeds of the outer cylinder, the SPT emerges through a supercritical scenario where simple secondary flows become unstable when increasing the inner cylinder speed. These instabilities lead to localized turbulent spots that eventually coalesce to form the SPT for higher inner rotations. For high speeds of the outer cylinder, the SPT regime is shown to be disconnected from other laminar solutions, retaining stability even when the base flow is linearly stable.

In Taylor-Couette flow, an incompressible fluid of kinematic viscosity $\nu$ and density $\varrho$ is contained between two concentric rotating cylinders whose inner and outer radii and angular velocities are $r_{i}^{*}, r_{o}^{*}$ and $\Omega_{i}, \Omega_{o}$ respectively. The dimensionless parameters are the radius ratio $\eta=r_{i}^{*} / r_{o}^{*}$ and the inner and outer Reynolds numbers $\mathrm{R}_{\mathrm{i}}=d r_{i}^{*} \Omega_{i} / \nu$ and $\mathrm{R}_{\mathrm{o}}=d r_{o}^{*} \Omega_{0} / \nu$ of the rotating cylinders. All variables are rendered dimensionless using the gap $d=r_{o}^{*}-r_{i}^{*}$ and viscous time $d^{2} / \nu$ as units for space and time, respectively. The dynamics of the flow is governed by the incompressible NavierStokes equations

$$
\partial_{t} \mathbf{v}+(\mathbf{v} \cdot \nabla) \mathbf{v}=-\nabla p+\Delta \mathbf{v}, \quad \nabla \cdot \mathbf{v}=0 .
$$

In nondimensional cylindrical coordinates $(r, \theta, z)$, the azimuthal circular Couette flow (CCF) is $\mathbf{v}_{B}=\left(u_{B}, v_{B}, w_{B}\right)=$ $(0, A r+B / r, 0)$, with $A$ and $B$ suitable constants so that $\mathbf{v}_{B}\left(r_{\mathrm{i}}\right)=\left(0, \mathrm{R}_{\mathrm{i}}, 0\right)$ and $\mathbf{v}_{B}\left(r_{\mathrm{o}}\right)=\left(0, \mathrm{R}_{\mathrm{o}}, 0\right)$ at the inner and outer radial walls $r_{\mathrm{i}}=\eta /(1-\eta)$ and $r_{\mathrm{o}}=1 /(1-\eta)$, respectively. The flow is assumed to be $L^{*}$-periodic in the axial direction so that the dimensionless domain is $(r, \theta, z) \in \mathfrak{D}=$ $\left[r_{i}, r_{o}\right] \times[0,2 \pi) \times[0, \Lambda)$, where $\Lambda=L^{*} / d$ is the aspect ratio of the computational box. The Navier-Stokes equations for arbitrary perturbations of the basic flow, $\mathbf{u}=\mathbf{v}-\mathbf{v}_{B}$, are discretised in space and time with a solenoidal spectral method that preserves zero net mass-flow in $z$ and with a 4th order linearly implicit time marching scheme, respectively [19]. Following former experimental works [7], the computations presented here were carried out for $\eta=0.883$, i.e., $r_{\mathrm{i}}=7.547$ and $r_{\mathrm{o}}=8.547$, with $\left(\mathrm{R}_{\mathrm{o}}, \mathrm{R}_{\mathrm{i}}\right) \in[-3000,-1200] \times[0,1000]$ and $\Lambda=29.9$. The spectral resolution used in our computations lies within the intervals $\left(N_{r}, N_{\theta}, N_{z}\right) \in 20 \times[100,220] \times$ $[100,220]$ radial $\times$ azimuthal $\times$ axial grid points, resulting in a dynamical system with $\mathcal{O}\left(10^{6}\right)$ degrees of freedom. In all cases shown, increasing the resolution did not provide noticeable changes.

Figure 1a shows angular momentum $\mathcal{L}=r v$ isosurfaces of SPT at $\left(\mathrm{R}_{\mathrm{O}}, \mathrm{R}_{\mathrm{i}}\right)=(-1200,600)$, conspicuously resembling the experimental results: $\mathcal{L}=1900$ (laminar) and $\mathcal{L}=-1900$ (turbulent) in white and blue, respectively. The two possible helical orientations were observed in our simulations with no apparent preference for any of them. Figure $1 \mathrm{~b}$ shows axial vorticity distribution on $z=0.83 \Lambda$ annular cross section (radially expanded for better visualization) within the range $(\nabla \times \mathbf{u})_{z} \in\left[-1.4 \times 10^{4}, 9.0 \times 10^{3}\right]$, with negative and positive vorticity regions in dark and light, respectively. The radial distribution of the SPT shows a clear trailing edge start-
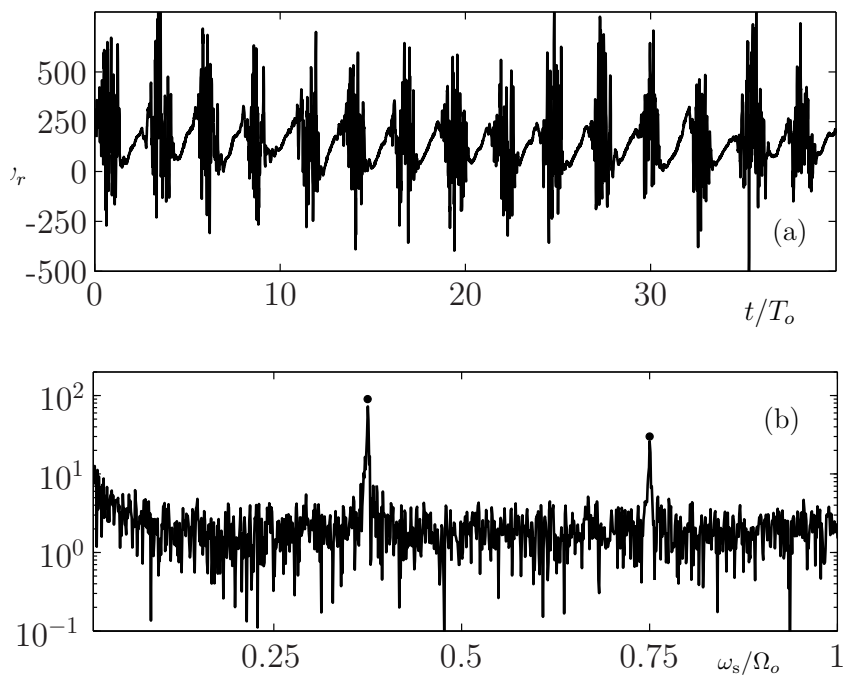

FIG. 2: Time series analysis of SPT for $\left(\mathrm{R}_{\mathrm{o}}, \mathrm{R}_{\mathrm{i}}\right)=(-3000,650)$. (a) Radial velocity measured at $(r, \theta, z)=(8.1,0,0)$ : time scale in $T_{o}=2 \pi / R_{\circ}(1-\eta)$ outer cylinder rotation period units. (b) Power spectra: frequency scale in $\Omega_{o}=\mathrm{R}_{\mathrm{o}}(1-\eta)$ outer cylinder angular speed units.

ing from the inner cylinder and progressively spreading outwards along half a perimeter until the leading edge is formed in the vicinity of the outer cylinder, as shown in Coles and Van Atta experiments [20]. Figure $1 \mathrm{~b}$ is a snapshot of a film showing spanwise (axial) vorticity filaments that are generated near the inner cylinder. However, contrary to centrifugal instabilities in rotating flows, these structures are not confined within the centrifugally unstable radial domain $r \in\left[r_{\mathrm{i}}, r_{\mathrm{n}}\right]$, with $r_{\mathrm{n}}=\sqrt{-B / A} \approx 7.867$ being the nodal radius, i.e., $v_{B}\left(r_{\mathrm{n}}\right)=0$. Moreover, these filaments are azimuthally driven by the outer cylinder, invading the whole radial domain and eventually detaching from the inner cylinder and breaking up near the outer wall. The observed phenomenon is remarkably similar to the usually termed as spike instability that appears in flat plate boundary layers [21].

Computed SPTs rotate with the same orientation as the outer cylinder and with a well defined phase speed independent of $\mathrm{R}_{\mathrm{i}}$. Figure 2 shows time series of radial velocity at a point of the domain and its corresponding spectral power analysis. The pseudoperiodicity of the signal is apparent just by bare eye inspection and the Fourier analysis reveals a fundamental frequency, corresponding to the phase speed $\omega_{\mathrm{S}}$ of the SPT, and its first harmonic.

The exploration reported here is summarised in the $\left(R_{0}, R_{i}\right)$ plane shown in Fig. 3. The linear stability boundary (LSB) corresponding to the theoretical $z$-invariant $\mathrm{CCF}$ may remarkably differ from the experimental transition thresholds for high values of $\mathrm{R}_{\mathrm{o}}$; nearly $20 \%$ for $\mathrm{R}_{\mathrm{o}}=-3000$ in this study. As already pointed out in [7], this discrepancy is ascribed to the axial distorsion of the azimuthal flow due to the pumping of fluid into the Ekman layers adjacent to the top and bottom lids. 


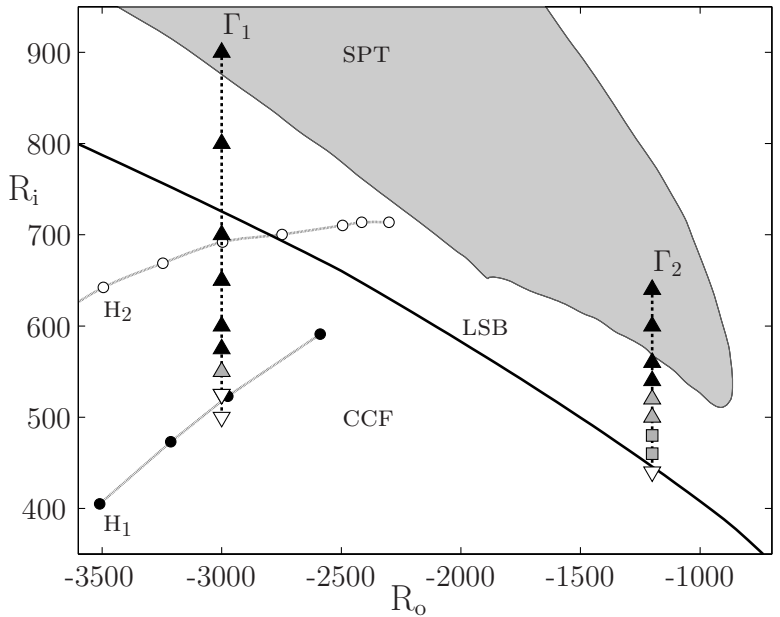

FIG. 3: Explored regions in $\left(\mathrm{R}_{\mathrm{o}}, \mathrm{R}_{\mathrm{i}}\right)$-parameter space. Black triangles, gray triangles, gray squares and white triangles correspond to SPT, INT, ISP and CCF flows, respectively. See text for explanation.

Two parametric paths for $\mathrm{R}_{\mathrm{O}}=-3000$ and $\mathrm{R}_{\mathrm{o}}=-1200$ (labelled as $\Gamma_{1}$ and $\Gamma_{2}$, respectively) were followed. Both paths start within the shadowed region of Fig. 3, where experiments [7] reported SPT regimes when increasing $R_{i}$ from rest. Starting with a random perturbation at $\left(\mathrm{R}_{\mathrm{O}}, \mathrm{R}_{\mathrm{i}}\right)=$ $(-3000,900)$ in $\Gamma_{1}$ and $\left(R_{\mathrm{o}}, \mathrm{R}_{\mathrm{i}}\right)=(-1200,640)$ in $\Gamma_{2}$, the time integrations drove the flow towards SPT patterns in less than one viscous time unit. From those starting points, $R_{i}$ was quasistatically decreased and the time evolution of the flow was monitored up to 10 viscous time units afterwards. Over $\Gamma_{2}$, SPT regimes followed exactly the same supercritical behaviour as the one observed in the experiments [7], where smooth decreasing of $R_{\mathrm{i}}$ sequentially led to intermittency regimes (INT, characterised by localised turbulent spots), interpenetrating spirals (ISP) and relaminarization to the basic CCF profile. However, over $\Gamma_{1}$, the SPT flow was found to be sustained even below the LSB curve. The $\mathrm{H}_{1}$ and $\mathrm{H}_{2}$ bulleted curves shown in Fig. 3 correspond to experimental hysteretic SPT boundaries when decreasing $\mathrm{R}_{\mathrm{i}}$ from above in [10] and [7], respectively. In particular, we found our computations to agree with the $\mathrm{H}_{1}$ boundary found by Coles, and this could be ascribed to the aspect ratio of the apparatus used in his experiments. The computations along the two paths have been repeated for smaller computational boxes $(\Lambda<20)$, although SPTs were never observed but uniform turbulent bursts appeared instead, in agreement with [16]. This means that the SPT needs a long enough apparatus to appear, but it is independent of the boundary conditions at the lids.

Fig. 4 outlines both explorations by showing the norm $<$ $E>^{1 / 2}$ corresponding to the time-averaged kinetic energy density of the observed regimes. In the subcritical case $\left(\mathrm{R}_{\mathrm{O}}=\right.$ $-3000)$, the SPT regime was observed within the range $R_{i} \in$ $[575,900]$, i.e., even below the linear instability threshold of CCF at $\mathrm{R}_{\mathrm{i}}=727.7$. However, for $\mathrm{R}_{\mathrm{i}}<575$, the SPT flow was no longer sustained and localised turbulent spots INT took

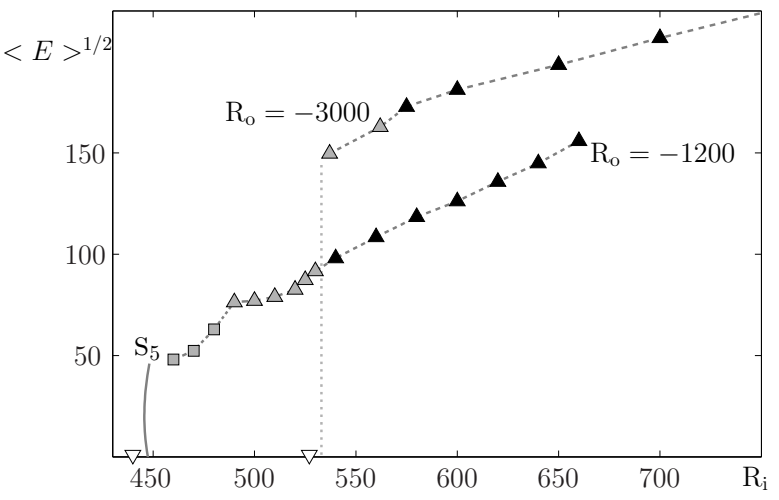

FIG. 4: Averaged norm $\langle E\rangle^{1 / 2}$ of the perturbation as a function of $R_{o}$ and $R_{i}$. The vertical dotted line of the top curve $R_{o}=-3000$ is located at the relaminarization value

over the dynamics within the range $\mathrm{R}_{\mathrm{i}} \in[537,562]$. Whereas the SPT regimes have a well defined mean energy value, the energy of the INT flow exhibits large oscillations and transient visits to regimes with large and small localised turbulent spots (see Fig. 5a and b).

In the supercritical scenario $\left(\mathrm{R}_{\mathrm{O}}=-1200\right)$, SPT flows were sustained for $\mathrm{R}_{\mathrm{i}} \in[540,640]$ (Fig. 5f), INT localised spots for $\mathrm{R}_{\mathrm{i}} \in[490,530]$ (Fig. 5d and e) and IPS flows for $\mathrm{R}_{\mathrm{i}} \in[450,480]$ (Fig. 5c). For $\mathrm{R}_{\mathrm{i}}<450$, the flow relaminarizes to the CCF basic flow, sometimes with a narrow interval of appearance of the recently found subcritical $\mathrm{S}_{5}$ spirals bifurcating from CCF at $\mathrm{R}_{\mathrm{i}}=447.4$ ( $\mathrm{S}_{5}$ gray curve in Fig. 4), with its saddle-node located at $\mathrm{R}_{\mathrm{i}}=445.7$, [17]. The transition from ISP to INT is quite abrupt. As soon as turbulent spots appear, no clear traces of ISP can be identified. Moreover, the angular advection of the involved flows changes drastically, i.e., whereas ISP are slowly advected by the inner cylinder, INT turbulent spots clearly follow the outer cylinder faster dynamics in the opposite direction. However, transition from INT to SPT was found to be smooth, whith a progressive helical alignment of the localised spots as long as $\mathrm{R}_{\mathrm{i}}$ increases. This sequence of transitions is clearly illustrated in Figs. 5c-f and their corresponding films.

To summarize, direct numerical simulation of SPT and localised turbulent spots in a small gap large aspect ratio TaylorCouette system has been carried out for the first time. These flows have been shown to require spanwise and streamwise extended domains to develop and that they do not critically depend on endwall effects from top and bottom lids. Computations have revealed that SPT originates at the inner cylinder by means of a detachment of spanwise vortex filaments that are advected by the outer cylinder, spreading out over the whole radial gap and eventually leading to a breakdown in the vicinity of the outer wall. This phenomenon clearly resembles other instability mechanisms already observed in flat plate boundary layer problems. Finally, the relation of the intermittent states to much simpler flows bifurcating from the base state has been evidenced at moderate counterrotat- 


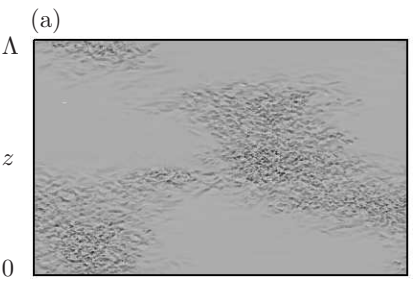

(b)
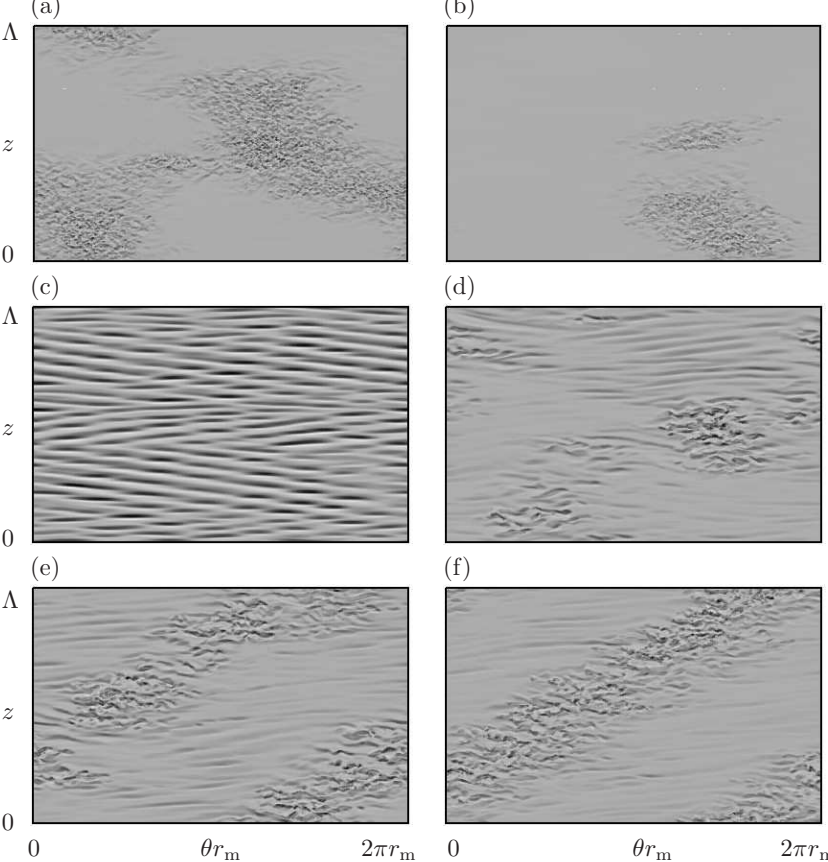

(d)

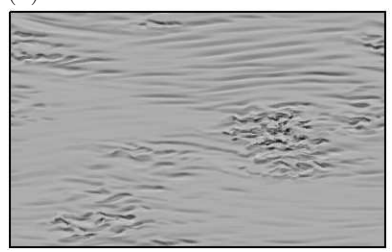

(f)

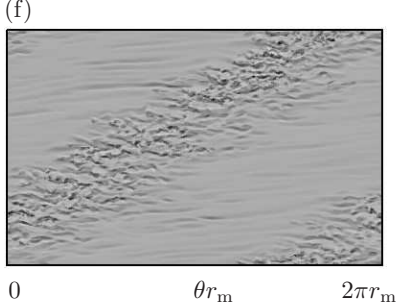

FIG. 5: Radial vorticity $(\nabla \times \mathbf{u})_{r}$ at intermediate radial surface $r_{m}=8.21$. (a) and (b) showing large and small turbulent spots for $\left(R_{o}, R_{i}\right)=(-3000,550)$. From (c) to $(d)$, same quantity for $\mathrm{R}_{\mathrm{o}}=-1200$. (c) ISP, $\mathrm{R}_{\mathrm{i}}=480$, (d) INT, $\mathrm{R}_{\mathrm{i}}=520$, (e) INT, $\mathrm{R}_{\mathrm{i}}=530$,(f) SPT, $\mathrm{R}_{\mathrm{i}}=600$ (see films).

ing speed. Higher speeds switch the bifurcating scenario from supercritical to subcritical, a unique feature of Taylor-Couette flow. This opens a promising path to understanding subcritical transition in other canonical shear flows that cannot be studied in the simpler frame of supercriticality.

AM, FMe and FMa research was supported by grants FIS2007-61585 and SGR-00024 from the spanish and cata- lan governments, respectively. MA work was funded by the Max Planck Society.

* Electronic address: alvarefa.upc.es

[1] A. Prigent, G. Gregoire, H. Chate, O. Dauchot, and W. van Saarloos, Phys. Rev. Lett. 89, 014501 (2002).

[2] D. Barkley and L. S. Tuckerman, Phys. Rev. Lett. 94, 014502 (2005).

[3] D. Barkley and L. S. Tuckerman, J. Fluid Mech. 576, 109 (2007).

[4] A. Darbyshire and T. Mullin, J. Fluid Mech. 289, 83 (1995).

[5] B. Hof, C. W. H. van Doorne, J. Westerweel, F. T. M. Nieuwstadt, H. Faisst, B. Eckhardt, H. Wedin, R. R. Kerswell, and F. Waleffe, Science 305, 1594 (2004).

[6] A. Willis and R. Kerswell, Phys. Rev. Lett. 100, 124501 (2008).

[7] C. D. Andereck, S. S. Liu, and H. L. Swinney, J. Fluid Mech. 164, 155 (1986).

[8] P. Chossat and G. Iooss, The Couette-Taylor Problem, App. Math. Sci. (Springer-Verlag, New York, 1994).

[9] R. Tagg, Nonlinear Sci. Today 4(3), 1 (1994).

[10] D. Coles, J. Fluid Mech. 21, 385 (1965).

[11] C. Van Atta, J. Fluid Mech. 25, 495 (1966).

[12] R. P. Feynman, Lecture Notes in Physics, vol. 2 (AddisonWesley, Reading, MA, 1964).

[13] J. J. Hegseth, C. D. Andereck, F. Hayot, and Y. Pomeau, Phys. Rev. Lett. 62(3), 257 (1989).

[14] A. Goharzadeh and I. Mutabazi, Eur. Phys. J. B 19, 157 (2001).

[15] A. Meseguer, Phys. Fluids 14(5), 1655 (2002).

[16] K. Coughlin and P. Marcus, Phys. Rev. Lett. 77(11), 2214 (1996).

[17] A. Meseguer, F. Mellibovsky, M. Avila, and F. Marques, Phys. Rev. E 79, 036309 (2009).

[18] H. Faisst and B. Eckhardt, Phys. Rev. E 61(6), 7227 (2000).

[19] A. Meseguer, M. Avila, F. Mellibovsky, and F. Marques, Eur. Phys. J. Special Topics 146, 249 (2007).

[20] D. Coles and C. W. Van Atta, Phys. Fluids Suppl. (1967).

[21] Y. S. Kachanov, Ann. Rev. Fluid Mech. 26, 411 (1994). 